\title{
Some Case Studies on Importance of Variables and Scales of Measurement in Social Sciences Research
}

\author{
Sahana Prasad \\ Associate Professor, Department of Statistics, Christ University, Bangalore, India
}

\begin{abstract}
Social Sciences studies human behaviour and it is very important that the conditions under which they occurred and also to describe the behaviour accurately and correctly. Thus it becomes important to have a "measure" of behaviour, description of all factors influencing behaviour and to what extent these influence such behaviour. They provide an opportunity for others to verify these observations. It is very important to present collected data in an accurate and easy-to-understand manner through various tools like diagrams and graphs. Every object possesses some characteristic which can be used as a scale for measurement. In the past, silver, food grains etc were used as units of measurement. concepts are large in number and part do not bear the kind of simple relationships to one another like in the physical sciences and there is a lack of strong mathematical theories against which to evaluate measurements in social sciences. A proper understanding of the scales of measurement which have to be used for different kinds of variables, is thus, an essential part of social sciences research. This paper focuses on some case studies in the usage of scales of measurement in social sciences research.
\end{abstract}

Keywords: Social Sciences research, variables, scales of measurement, case studies

\section{INTRODUCTION}

While evaluating information about the social world about any particular phenomenon, say $\mathrm{X}$, it is important to focus on two aspects:

"What is meant by $\mathrm{X}$ in this research?"

For instance, if a researcher is measuring satisfaction level of employees, it is essential to define and bear in mind what this means. Is it the mental state when they leave work place? Or the mind set when they step in? or their perception about workplace?

"How was X measured?"

Which are the scale/s that is helpful in measuring the phenomenon under study?

Almost all research in social science research involves a process in which a researcher takes a general concept or idea, specifies the dimensions that he/she wants to study, then creates measures to evaluate these dimensions. These are the processes known as conceptualization and operationalization. Singleton and Straits (1999) refer to the process of establishing a conceptual definition in addressing the first of these questions, while addressing the second is the creation of an operational definition.

Every object possesses some characteristic which can be used as a scale for measurement. In the past, silver, food grains etc were used as units of measurement. concepts are large in number and part do not bear the kind of simple relationships to one another like in the physical sciences and there is a lack of strong mathematical theories against which to evaluate measurements in social sciences. Social scientists have used "subject-centred measurement" which is typically done through the use of Likert scales with response categories such as "Definitely disagree," “disagree," "neutral," "agree," and "definitely agree"). There are other scales like "stimulus centred measurement" where one orders stimuli from high to low on a scale. Some other examples include "Behaviourally anchored rating scales (BARS)", semantic differential, Guttman scale and many more.

S.S. Stevens in his paper "On the theory of scales of measurement" defines four hierarchical scales of measurement namely nominal, ordinal, interval and ratio scale. Though there are many discussions on the pros and cons of this classification, it is widely used in research. Its importance is due to the fact that the choice of an appropriate model for analysis depends on this level of measurement and one point to be kept in mind is that the higher the level of measurement, more information is available about the variable.

\section{Scales of Measurement}

Nominal or classificatory scale is the simplest level wherein objects are categorized into different categories which are exclusive as well as exhaustive. It means that one observation can be put under only one category and there should be enough categories to put in all observations. These categories, which have to be nonoverlapping, are generally called attributes. An easy example would be classification of population as male and female. They can be given codes like 0,1 which indicate their gender. It has to be clearly understood that they do not mean that 0 is less than 1 . The codes are meant only for classification and understandability. It is also important to note that though a unit has many characteristics, the researcher focuses only on that which is relevant to the study. Also, the symbols or codes used can be interchanged or altered without causing any alterations in the data.

What to use: Mode, proportion, frequency, chi-square tests, contingency coefficient are those statistical operations which can used for nominal data. 
Ordinal scale is used to indicate the place of a category in an ordered series and they state the position of the characteristic with respect to others. Thus, in an ordinal scale, the values are hierarchical and state the relative position of a characteristic and unlike nominal scales, ordinal scales allow comparisons of the degree to which two subjects possess the characteristic. Generally, the number 1 is assigned to that which has larger quantity of what is being studied just like a student with the highest marks gets the first rank. The others are ranked according to their marks. It has to be remembered though that the difference between two levels of an ordinal scale is not the same as the difference between two other levels. For e.g.: If 1 denotes highly satisfied, 2 denotes satisfied and 3 denotes moderately satisfied, 2-1 is not the same as 3-2.

What to use: Median, Quartile deviation, Spearman's rank correlation, Mann-Whitney U test are some of the valid tools for ordinal data.

Interval Scale is the scale having all properties of nominal as well as ordinal scale. In addition, it has the characteristics of equal intervals, which means that the difference between 40 degrees $F$ and 50 degrees $F$ represents the same temperature difference as the difference between 80 degrees and 90 degrees. This is because each 10-degree interval has the same physical meaning. This scale has a true zero point even if one of the scaled values is called "zero." The Fahrenheit scale is an example for this. Zero degrees Fahrenheit does not mean absence of temperature it does not make sense to compute ratios of temperatures. Also, there is no sense in which the ratio of 40 to 20 degrees Fahrenheit is the same as the ratio of 100 to 50 degrees. If, for instance, "zero" label was applied at the temperature 10 degrees, the two ratios would instead be 30 to 10 and 90 to 40.Thus, it does not right to say that 80 degrees is "twice as hot" as 40 degrees.

What to use: The tools and operations in interval scale can be AM, median, SD, product moment correlation coefficient and other parametric tests like z, t, F etc

Ratio Scale is the most informative scale. It is an interval scale with the additional property that its zero position indicates the absence of the quantity being measured. Ratio scale can be regarded as the three earlier scales rolled up in one. Like a nominal scale, it provides a name or category for each object (the numbers serve as labels). Like an ordinal scale, the objects are ordered and like an interval scale, the same difference at two places on the scale has the same meaning. And in addition, the same ratio at two places on the scale also carries the same meaning. The Fahrenheit scale for temperature has an arbitrary zero point and is therefore not a ratio scale. However, zero on the Kelvin scale is absolute zero. This makes the Kelvin scale a ratio scale. Similarly, money is measured on a ratio scale because, in addition to having the properties of an interval scale, it has a true zero point: if one has zero money, this implies the absence of money. Since money has a true zero point, it is correct to say that someone with 50 rupees has twice as much money as someone with 25 rupees.
What to use: Any statistical tool, like GM, CV etc which requires knowledge of true scores can be used. Also, any statistical test-parametric or non parametric can be used.

1) Contextual cases

Case I: Consider a research in which a questionnaire is administered on their opinion on "Capital punishment ". The ratings maybe on a 5point scale starting with 1 as highly agree.

Right Tool: Ordinal Scale

Reason: Ordinal Scale is the right choice as there is a hierarchy but the difference between 1 and 2 doesn't mean the same between 3 and 4 .

Case II: Consider a set of clothes 1: socks 2: shirt 3: trousers 4: handkerchief

Right Tool: Nominal Scale

Reason: The scale is nominal as these numbers are only used for identification purposes only.

Consequences of choosing the wrong level of

measurement

The relationship between the variable's level of measurement and the statistics that can be meaningfully computed with that variable is very important. For example, consider a hypothetical study in which 10 girls are asked to choose their favourite colour of scarves from blue, red, yellow, green, and white. The researcher codes the results as follows:

\begin{tabular}{|l|c|c|}
\hline Subject & Colour & Code \\
\hline 1 & Blue & 1 \\
\hline 2 & Red & 2 \\
\hline 3 & Green & 4 \\
\hline 4 & White & 5 \\
\hline 5 & White & 5 \\
\hline 6 & Yellow & 3 \\
\hline 7 & Yellow & 3 \\
\hline 8 & Green & 4 \\
\hline 9 & Red & 2 \\
\hline 10 & White & 3 \\
\hline
\end{tabular}

This means that if a girl said her favourite colour was "Red," then the choice was coded as "2," if she said her favourite colour was "White," then the response was coded as 5 , and so on. Consider the following hypothetical data Since each code is a number, we may feel like computing the average code assigned to the girls. The average is 3.5, and the conclusion would be that the average favourite colour is between yellow and green. It can be clearly seen that it is a meaningless conclusion.

\begin{tabular}{|l|c|c|}
\hline Subject & Colour & Code \\
\hline 1 & Blue & 1 \\
\hline 2 & Red & 2 \\
\hline 3 & Green & 4 \\
\hline 4 & White & 5 \\
\hline 5 & White & 5 \\
\hline 6 & Yellow & 3 \\
\hline 7 & Yellow & 3 \\
\hline 8 & Green & 4 \\
\hline 9 & Red & 2 \\
\hline 10 & White & 3 \\
\hline
\end{tabular}


How to relate

If the study is about comparison of two independent groups, e.g. Birth weights of babies from a group of South Indian mothers compared with those from a group of North Indian mothers.

We can use nominal data to just represent the babies in different birth weights and then proceed to Chisquare test

If ordinal scale is used, Mann- Whitney U test can be applied.

If it is dichotomous, that is has only 2 cases, we can use Fisher's exact test.

It interval or ratio scale is used; we have to take sample size also into consideration.

i. If the sample size is large ( $\mathrm{n}>100)$, we can use $\mathrm{z}$ (normal distribution) test for the comparison of means.

ii. If the sample is small to medium drawn from a normal distribution, we can use unpaired (two sample) student $t$ test

iii. If the sample is small to medium drawn from a normal distribution, we can use the Mann- Whitney U test

Note: In the social and behavioural sciences, characteristics are measured on an ordinal level. We often ask if people "Strongly disagree" till "Slightly Disagree". We then assign a value of '1' if they strongly disagree with a statement, up to a '5' if they strongly agree with a statement. This type of measurement is ordinal as "Strongly Agree" reflects more agreement than "Slightly Agree". This type of measurement is not an interval or a ratio level of measurement, because we cannot state with certainty that the interval between "Strongly Disagree" and "Slightly Disagree" is equivalent to the interval between "Slightly Disagree" and "Neutral". Also, we say that there is an absolute zero point for level of agreement. But it has been found that for the many studies, it is not wrong to treat ordinal data (such as variables which have been measured using Strongly Disagree to Strongly Agree response alternatives) as interval level data, and conduct statistical tests that are appropriate for interval level data.

Example: Consider a set of data values obtained through a questionnaire along with their levels of measurement

Data 1: Name of respondent

Level of measurement: Nominal

Why? Because age does not indicate any order and it is just identification.

Data 2: Age of respondent

Level of measurement: Ratio

Why? Because age has a meaningful zero. It is relevant to find ratio, for example, a 30yr old person is twice as old as a 15 yr old.

Data 3: Which year did you finish secondary, senior secondary and degree exams?

Level of measurement: Interval

Why? Data can be ordered and difference in data has a meaning. For e .g, 2004 comes 4 yrs later than 2000. But 2004 is not twice that of 1002 . Also, "0" does not mean "absence of time".
Data 4: What was the total tax paid by you last financial year?

Level of measurement: Ratio

Why? If a person paid Rs. 10,000 as tax, it is half of what someone else who paid Rs. 20,000

Data 5: How do you rate the new serial which is being telecast

8pm on "Stupid TV"? Choices were (i) very stupid

(ii) quite stupid

(iii) somewhat stupid

(iv) not stupid

Level of measurement: Ordinal

Why? The choices can be ordered but there are no meaningful numerical differences between them. For instance, "quite stupid" is not double of "somewhat stupid"

Data 5: What is your marital status? 1- Unmarried or 2married

Level of measurement: Nominal

Why? These numbers 1 and 2 serve as indicators only

A note on validity and reliability: To evaluate and measure or observation, one needs to check:

Q1: Are we are measuring what we intend to measure? Validity

Q2: Will the same measurement process yield the same results when the process is repeated? reliability.

As a researcher, one needs measurement tools that are both reliable and valid. In other words, the researcher wants questions that yield consistent responses when asked multiple times (reliability). As also, questions that get accurate responses from respondents (validity).

\section{CONCLUSION}

Classification of variables according to their measurement scales is useful to help in choosing an analytic procedure but it cannot be a substitute for using sound judgment when choosing a statistical analysis.

Many statistical methods are appropriate only for data of certain measurement scales. When selecting a statistical method, it is essential to understand how the data to be analyzed were measured. At the design stage itself one needs to understand the statistical limitations imposed by certain measurement scales and how they can influence choice of observations and methods of measurement.

\section{ACKNOWLEDGMENT}

I acknowledge the support of Centre for Research, Christ University for preparation of monograph "Applications of Statistical Tools in Social Sciences Research" from which this paper is derived.

\section{REFERENCES}

[1] B Y Brase, C. H. (n.d.). Understanding basic statistics

[2] Healey, J. (n.d.). Statistics- A tool for Social Research. Newport University.

[3] Linneman, T. J. (n.d.). Social Statistics-Basics and Beyond

[4] P.K.Majumdar. (n.d.). Statistics-A tool for Social Sciences Utts, J. H. (n.d.). Mind on Statistics.

6] http://onlinestatbook.com/2/introduction/

levels_of measurement.html 
International Advanced Research Journal in Science, Engineering and Technology

Vol. 2, Issue 3, March 2015

\section{BIOGRAPHY}

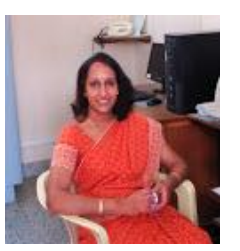

Mrs. Sahana Prasad has been teaching Statistics from the past 26 years and has published a monograph on "Application of Statistical Tools in Social Sciences Research". She also has published a large number of articles and stories in Kannada and English newspapers and magazines. She has helped many researchers with statistical consultancy 